\title{
The Occurrence and Longitudinal Changes of Cognitive Impairment After Acute Ischemic Stroke
}

This article was published in the following Dove Press journal:

Neuropsychiatric Disease and Treatment

\author{
Xiao-ling Liao' \\ Li-jun Zuo' \\ Ning Zhang ${ }^{2}$ \\ Yang Yang ${ }^{2}$ \\ Yue-song $\mathrm{Pan}^{3}$ \\ Xiang-long Xiang ${ }^{3}$ \\ Ling-yun Chen ${ }^{4}$ \\ Xia Meng ${ }^{3}$ \\ $\mathrm{HaO} \mathrm{Li}^{3}$ \\ Xing-quan Zhao (1) \\ Yi-long Wang' \\ Chun-xue Wang ${ }^{2}$ \\ Jiong $\mathrm{Shi}^{3}$ \\ Yong-jun Wang'
}

On behalf of the Impairment of CognitiON and Sleep quality for patients after acute ischemic stroke or transient ischemic attack (ICONS) Investigators

'Department of Neurology, Beijing Tiantan Hospital, Capital Medical University, Beijing, People's Republic of China; ${ }^{2}$ Department of Neuropsychology, Beijing Tiantan Hospital, Capital Medical University, Beijing, People's Republic of China; ${ }^{3}$ National Clinical

Research Center for Neurological Diseases, Beijing Tiantan Hospital, Capital Medical University, Beijing, People's Republic of China; ${ }^{4}$ Department of Medicine, Eisai China INC, Shanghai, People's Republic of China

Correspondence: Yong-jun Wang Department of Neurology, Beijing Tiantan Hospital, Capital Medical University, No. I I9, South Fourth Ring West Road, Fengtai District, Beijing 100070, People's Republic of China

Tel +86-10-59978350

Fax +86-10-59973383

Email yongjunwang@ncrend.org.cn
Background and Purpose: More and more evidence suggests that cognitive impairment (CI) after stroke is closely related to the quality of life of stroke patients. The primary aim of this study is to investigate the occurrence and longitudinal changes of $\mathrm{CI}$ at different stages after acute ischemic stroke (AIS) in Chinese patients.

Methods: The data of this study come from the impairment of cognition and Sleep after acute ischemic stroke or transient ischemic attack in Chinese patients study (ICONS), a nationwide multicenter prospective registry that recruited consecutive AIS or transient ischemic attack inhospital patients within 7 days after onset. Patients were followed for Montreal Cognitive Assessment (MoCA) scale at 2-week (2w), 3 months (3m) and 12 months (12m). CI was defined as MoCA score $\leqq 22$. No cognitive impairment (NCI) was defined as MoCA score $>22$.

Results: A total of 2432 AIS patients were enrolled in this study. Overall, $72.94 \%$ of patients were male and the average age was 60.95 years. Median National Institutes of Health Stroke Scale score was 3. The occurrence rate of CI was $52.38 \%, 35.55 \%$ and $34.16 \%$ at $2 \mathrm{w}, 3 \mathrm{~m}$ and $12 \mathrm{~m}$. Among patients with $\mathrm{CI}$ at $2 \mathrm{w}$ and $3 \mathrm{~m}, 39.9 \%$ and $27.9 \%$ of patients returned to NCI at next follow-up point. At $3 \mathrm{~m}$ and $12 \mathrm{~m}$ follow-up, there were also $9.6 \%$ and $12.7 \%$ new CI patients. The two cognitive items with the highest abnormal rate were "Delayed recall" (89.35\%, 83.33\% and 82.80\%) and "Visuospatial/executive" (78.91\%, $73.42 \%$ and $70.08 \%$ ). The cognitive item with the highest percentage of improved patients was "Orientation" (60.91-76.68\%), and the cognitive item with the lowest percentage of improved patients was "Language" (35.85-44.50\%).

Conclusion: CI had a relatively high occurrence at $2 \mathrm{w}$ to $12 \mathrm{~m}$ after AIS. CI at $3 \mathrm{~m}$ and $12 \mathrm{~m}$ was significantly lower than that at $2 \mathrm{w}$ after stroke. The occurrence of abnormalities and recovery probability for different cognitive items also differed greatly.

Keywords: cognitive impairment, ischemic stroke, longitudinal cohort study, occurrence

\section{Introduction}

Stroke is the second leading cause of death worldwide and the leading cause of mortality and disability in China. ${ }^{1,2}$ Research and interventions have historically mainly focused on physical disabilities, but more and more evidence suggests that cognitive impairment $(\mathrm{CI})$ is closely related to the prognosis of stroke patients. ${ }^{3-5}$ Poststroke cognitive impairment (PSCI) may reduce the quality of life, and increase the risk of stroke recurrence. ${ }^{6}$ It cast a heavy economic burden to families and the society. PSCI has gained more attention in current stroke research and intervention internationally. In American Heart Association (AHA)/American Stroke Association (ASA) 2016 guidelines for adult stroke rehabilitation and recovery, screening for cognitive deficits is recommended for all stroke patients before discharge. ${ }^{7}$ 
Stroke burden in China has increased over the past 30 years. There are 7 million stroke patients in China. The age-standardized occurrence is $246.8 / 100000$ personyears. ${ }^{8,9}$ However, the public awareness of CI after stroke is low in China. There is currently no nationwide multicenter survey of CI in acute stroke patients in China, except data from local cities.

The primary aim of this study is to investigate the occurrence and longitudinal changes of $\mathrm{CI}$ at different stages after acute ischemic stroke (AIS) in Chinese patients.

\section{Methods}

\section{Overview of the Study}

The data of this study come from the Impairment of CognitiON and Sleep after acute ischemic stroke or transient ischemic attack in Chinese patients (ICONS) study. In this manuscript, we analyzed the occurrence and longitudinal changes of CI after AIS. ICONS is one of the research subgroups of China National Stroke Registry-III (CNSR-III). ${ }^{10}$ CNSR-III is a nationwide prospective registry for consecutive patients presented to hospitals within 7 days after AIS or transient ischemic attack (TIA) between August 2015 and March 2018 in China. The protocol of the CNSR-III study was approved by ethics committee at Beijing Tiantan Hospital. Written informed consent was obtained from all patients or his/her representatives before being entered into the study.

The study sites with the experience of cognition and sleep research among CNSR-III were selected to participate in the ICONS study. Among the patients of CNSR-III who met the sub-group inclusion criteria were selected and included in ICONS. The main aims of ICONS are to investigate the occurrence and the influencing factors of $\mathrm{CI}$ and sleep disorder at 2-week (2w), 3-month (3m), and 12-month $(12 \mathrm{~m})$ after AIS/TIA in Chinese patients. The protocol of the ICONS study was also approved by ethics committee at Beijing Tiantan Hospital and all participating centers. Separate written informed consent was also obtained. Both studies were conducted in accordance with the Declaration of Helsinki.

\section{Site Selection}

The project of ICONS was carried out in multiple hospitals and medical centers. The steering committee hospitals nationwide to represent the population from each region of East, West, South, North, Center of Mainland China. 52 hospitals were invited initially, 46 agreed, and 40 were ultimately selected by the ICONS steering committee based on their research capability and commitment to the study. Figure 1 presents the geographical locations of these 40 participating hospitals.

\section{Patient Enrollment and Definition of $\mathrm{Cl}$}

The ICONS study recruited consecutive patients from August 2015 to January 2018 who met the following criteria:

\section{Inclusion Criteria}

1. Age $\geq 18$ years old;

2. Diagnosis of AIS/TIA;

3. Admission within 7 days after onset of AIS/TIA.

4. Ability to understand, confirm and sign the informed consent.

\section{Exclusion Criteria}

1. Silent cerebral infarction diagnosed by MRI or CT without symptoms and signs.

2. Illiterate patients.

3. History of cognitive impairment, psychosis or schizophrenia disease (documented in medical records).

4. Other factors that interfere with cognitive or sleep evaluation, e.g. severe aphasia defined as National Institutes of Health Stroke Scale (NIHSS) item 9 (Best Language) $>2$, consciousness disorders defined as NIHSS item 1a (Level of Consciousness) $>1$ or $1 \mathrm{~b}$ (LOC Questions) $>1$, hearing loss, visual impairment, hard to cooperate, severe unilateral neglect or dyslexia.

5. Unable to complete the baseline Montreal Cognitive Assessment (MoCA) and other tests.

Acute ischaemic stroke and TIA were diagnosed according to the WHO criteria (acute onset of neurological deficit, persisting for $>24$ hours in case of stroke, or for $<24$ hours in case of TIA) and confirmed by MRI or brain CT (If with evidence of associated acute focal infarction on imaging, the patient will be diagnosed as a stroke rather than a TIA, even if neurological deficit persisting for $<24$ hours). ${ }^{11,12}$ Considering the difference in duration of symptoms and infarction between TIA and AIS patients, the cognitive abnormalities of them will be different. In this study, we only choose the AIS population for analysis.

In this study, CI was defined as MoCA $\leqq 22$. No cognitive impairment (NCI) was defined as MoCA score $>22$. 


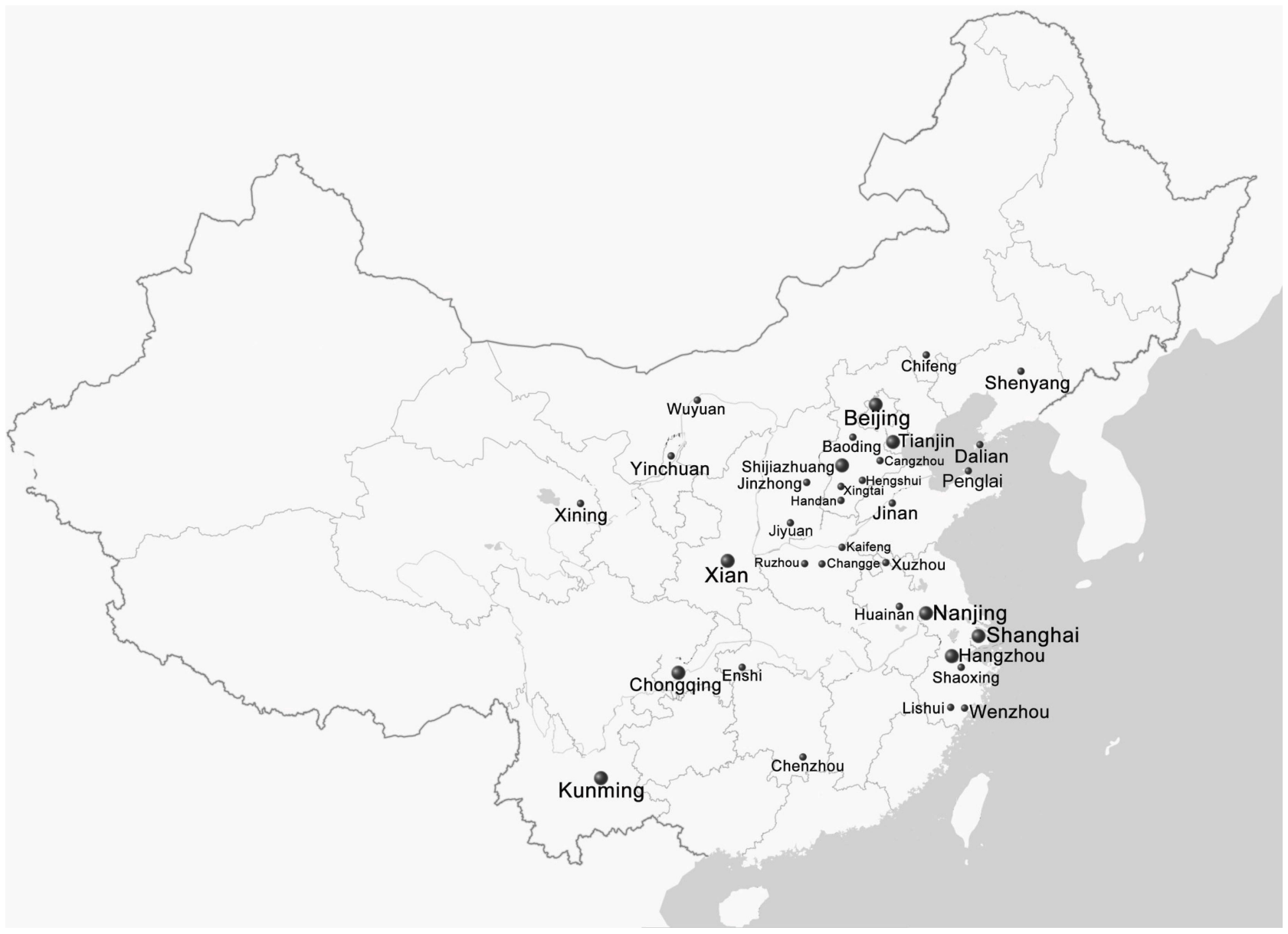

Figure I The geographical locations of participating hospitals in the ICONS.

Regarding the identification of PSCI, the best cut-off point was still be controversial. ${ }^{13-16}$ The meta-analysis by Lees et al showed that the conventional cut-off point $(<26 / 30)$ had excellent sensitivity (0.95) but suboptimal specificity (0.45). By comparison, the adapted MoCA cut-off point $(<22 / 30)$ improved specificity $(0.78)$ while maintaining good sensitivity (0.84). ${ }^{15}$ Previous research by our team also found that MoCA cut-off score of 22/23 was optimally sensitive $(0.85)$ and specific $(0.88)$ for detecting CI after mild stroke and TIA in Chinese patients. ${ }^{16}$ The overall patients enrolled in our study may be relatively mild. So in this study, we also define $\mathrm{CI}$ as $\mathrm{MoCA} \leqq 22$.

\section{Baseline/Follow-Up Data Collection and Data Management}

The ICONS study baseline data and follow-up collection and processing methods were the same as the CNSR-III study. ${ }^{10}$ Patients were interviewed face-to-face at $2 \mathrm{w}, 3 \mathrm{~m}$ and $12 \mathrm{~m}$ and contacted over the telephone by trained research coordinators at $6 \mathrm{~m}$ and 2-5 years annually.

Based on the follow-up content of CNSR-III study. For all patients included in the ICONS study, at $2 \mathrm{w}$ or discharge, $3 \mathrm{~m}$ and $12 \mathrm{~m}$, MoCA, ${ }^{13,17}$ Pittsburgh Sleep Quality Index (PSQI), ${ }^{18}$ Epworth Sleeping Scale (ESS), ${ }^{19}$ Patient Health Questionnaire-9 (PHQ-9), ${ }^{20}$ General Anxiety Disorder-7 (GAD-7), ${ }^{21}$ 10-meter walking speed test and stroke impact scale (SIS-16) were evaluated face to face. ${ }^{22,23}$ All tests were administered with same version by trained examiners. The medications for cognitive impairment, sleep disorder and depression were also recorded.

The MoCA scale is the most commonly used cognitive instrument for screening for $\mathrm{CI}$ in patients with mild stroke or TIA. It is a one-page 30-point test for cognition administered in about 10 mins, including Visuospatial/executive abnormal (5 points), Naming (3 points), Attention (2 points), Language ( 2 points), Delayed recall ( 5 points) Orientation ( 6 points). ${ }^{13,17}$ The same version of MoCA were used at all follow-up point. 


\section{Statistical Analysis}

Means with standard deviation or median with the interquartile range were used for continuous variables. Proportions were used to describe the categorical variables. $t$-test or Mann-Whitney test was performed to compare continuous variables, while as chi-square test or fisher's exact test was performed to compare the categorical variables. A two-sided $\mathrm{p}$ value $<0.05$ was considered to be statistically significant. All analyses were conducted with SAS 9.4 (SAS Institute Inc, Cary, NC).

\section{Results}

Patient recruitment and $12 \mathrm{~m}$ follow-up had been completed. There were 2704 patients who were screened and consented from 40 participating sites between August 2015 and January 2018. Finally, a total of 2625 patients were eligible for ICONS study. 2432 AIS patients were included for this study. The detailed patient enrollment flow chart is shown in Figure 2.

Baseline characteristics of the included AIS patients are presented in Table 1. Overall, 72.94\% of patients were male and the average age was 60.95 years. The median time from disease onset to enrollment was 1 day. Median NIHSS score was 3.
Number of completed people for face-to-face evaluation of $\mathrm{MoCA}$ and the occurrence rate of $\mathrm{CI}$ at each follow-up point is shown in Table 2. The occurrence rate of $\mathrm{CI}$ (defined as $\mathrm{MoCA} \leqq 22$ ) was $52.38 \%, 35.55 \%$ and $34.16 \%$ at $2 \mathrm{w}, 3 \mathrm{~m}$ and $12 \mathrm{~m}$. At the three follow-up time points $(2 \mathrm{w}, 3 \mathrm{~m}$ and $12 \mathrm{~m})$, the two cognitive items with the highest abnormal rate were "Delayed recall" (89.35\%, $83.33 \%$ and $82.80 \%$ ) and "Visuospatial/executive" (78.91\%, 73.42\% and 70.08\%).

Figure 3 details the longitudinal changes of CI (defined as $\mathrm{MoCA} \leqq 22$ ) at each follow-up point. Among patients with $\mathrm{CI}$ at $2 \mathrm{w}$ and completed MoCA follow-up at $3 \mathrm{~m}$, $39.9 \%(433 / 1084)$ of patients returned to NCI at $3 \mathrm{~m}$ follow-up. And among patients with CI at $3 \mathrm{~m}$ and completed MoCA follow-up at 12m, 27.9\% (127/456) patients returned to NCI at $12 \mathrm{~m}$ follow-up. At $3 \mathrm{~m}$ and $12 \mathrm{~m}$ followup, there were also 9.6\% (98/1023) and 12.7\% (105/828) new CI patients.

Table 3 shows the percentage of improved patients for each abnormal cognitive item in MoCA scale at $3 \mathrm{~m}$ and $12 \mathrm{~m}$ follow-up compared with those at $2 \mathrm{w}$ or $3 \mathrm{~m}$. The cognitive item with the highest percentage of improved patients was "Orientation" (60.91-76.68\%), and the cognitive item with the lowest percentage of improved patients was "Language" (35.85-44.50\%).

\section{4 patients consented and enrolled}

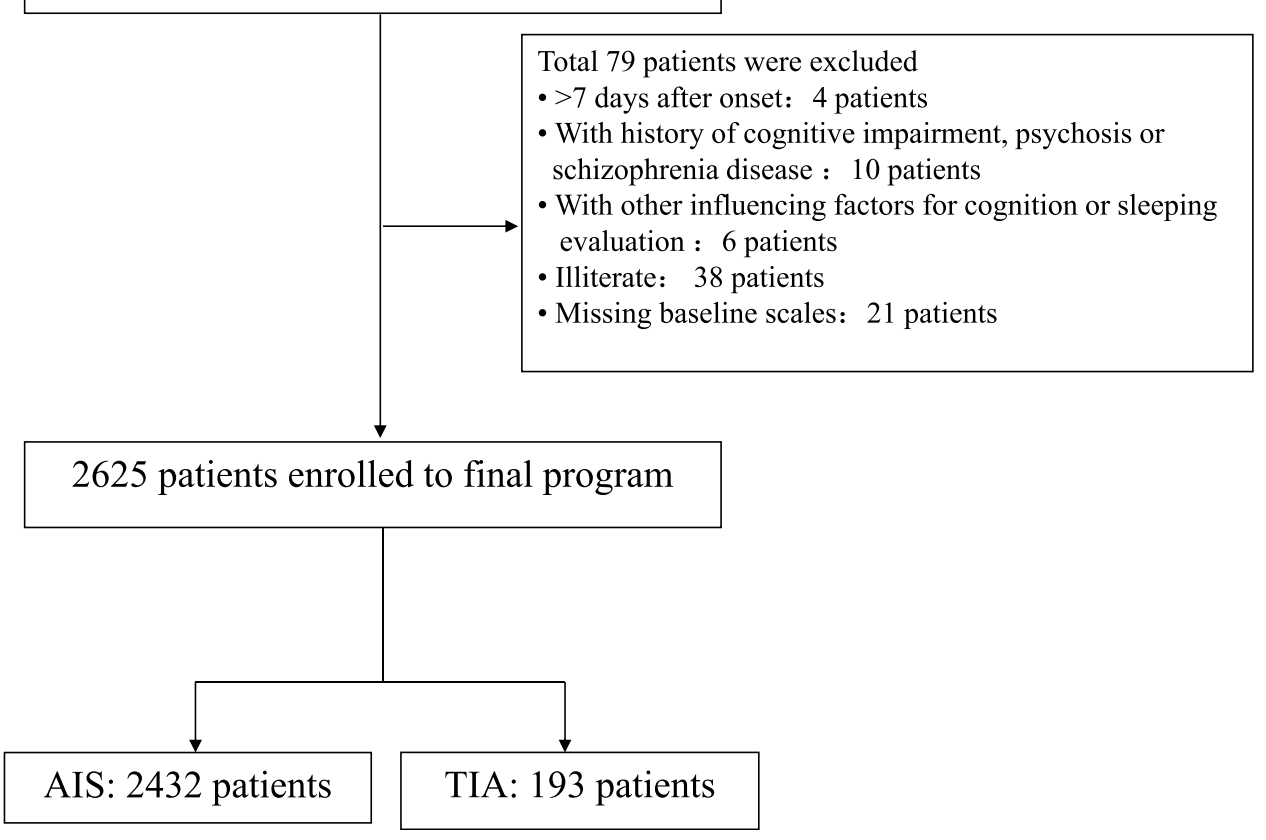

Figure 2 Flow chart of patient enrollment in the study. There were 2704 patients who were screened and consented from 40 participating sites. Finally, a total of 2432 acute ischemic stroke patients were included for this study. 
Table I Baseline Demographic Characteristics, Medical History and Stroke Subtype

\begin{tabular}{|c|c|}
\hline Baseline Variables & AIS $n(\%) ~ N=2432$ \\
\hline Gender male & I774(72.94) \\
\hline Average age (years) & $60.95 \pm 10.78$ \\
\hline Race (Han) & $235 I(96.67)$ \\
\hline \multicolumn{2}{|l|}{ Education level } \\
\hline Elementary or below & $638(26.23)$ \\
\hline Middle school & $867(35.65)$ \\
\hline High school or above & $809(33.26)$ \\
\hline Unknown & $118(4.85)$ \\
\hline Current smoker & $890(36.60)$ \\
\hline Secondhand smoking & $385(15.83)$ \\
\hline Heavy drinker $(>60 \mathrm{~g} / \mathrm{d})$ & $154(6.33)$ \\
\hline \multicolumn{2}{|l|}{ Medical History } \\
\hline Diabetes & $562(23.11)$ \\
\hline Hypertension & $1530(62.91)$ \\
\hline Lipid metabolism disorders & $234(9.62)$ \\
\hline Cerebral infarction & $509(20.93)$ \\
\hline $\mathrm{ICH}$ & $32(1.32)$ \\
\hline Subarachnoid hemorrhage & $4(0.16)$ \\
\hline TIA & $64(2.63)$ \\
\hline Heart failure & $9(0.37)$ \\
\hline Atrial fibrillation & $72(2.96)$ \\
\hline Carotid artery stenosis & $15(0.62)$ \\
\hline Epilepsy & $7(0.29)$ \\
\hline Sleep Apnea & $29(1.19)$ \\
\hline Median NIHSS score (IQR) & $3(I-5)$ \\
\hline \multicolumn{2}{|l|}{ Stroke subtype for TOAST } \\
\hline Large artery atherosclerosis & $1407(57.85)$ \\
\hline Cardiogenic embolism & $99(4.07)$ \\
\hline Small artery occlusion & $768(31.58)$ \\
\hline Other/Unknown & $158(6.50)$ \\
\hline Median Stroke onset to enrollment time (day) & $I(0-2)$ \\
\hline
\end{tabular}

Abbreviations: ICH, Intracranial Cerebral Hemorrhage; TIA, Transient Ischemic Attack; NIHSS, National Institutes of Health Stroke Scale; IQR, interquartile range; TOAST, the Trial of Org 10,172 in Acute Stroke Treatment.

\section{Discussion}

ICONS is the first prospective national registry study for cognitive impairment and sleep disorders on AIS and TIA in an inpatient population in China. In this manuscript, we mainly introduced the overall protocol of ICONS and analyzed the occurrence and longitudinal changes of $\mathrm{CI}$ after AIS.

The occurrence of CI after stroke in previous studies varies greatly depending on the region, ethnicity, diagnostic criteria, stroke severity, assessing time after stroke, and the method of assessment. The MoCA and the Mini-
Mental State Examination (MMSE) were commonly recommended tools for screening for CI. They include relatively good global cognitive assessment and the operation is relatively simple.

In European countries, using the MMSE or MoCA, the occurrence of $\mathrm{CI}$ at 3 months after stroke was $24 \%$ to $47 \%{ }^{24,25}$ However, using comprehensive neuropsychological tests which is more comprehensive and accurate for cognitive function evaluation, the occurrence of $\mathrm{CI}$ in the same population may be higher ( $96 \%$ vs $39 \%) .{ }^{25}$ In Asia, a multicenter, cohort study in Korea that enrolled 620 patients with ischemic stroke and used MMSE assessment showed that the occurrence of CI was as high as $69.8 \%$ at 3 months after stroke. ${ }^{26}$ In China, a study of ischemic stroke patients in Chongqing city, using MMSE assessment, the occurrence of CI after 3 months of stroke was $32.2 \% .^{27}$

Most of the reported PSCI studies only followed patients up to 3 months after stroke. Since cognitive decline is usually a long-term process, a longer follow-up period is warranted. The ICONS study was the first prospective national registry study for CI after AIS and TIA in an inpatient population in China. Compared with previous studies, ICONS study has more frequent and longer follow-ups. According to the inclusion criteria of this study, some of more severe populations were not selected in this study because of the difficulty in accurately evaluating MoCA. Therefore, the selected population in this study was actually younger and milder than the typical stroke population, but the results showed that there was still a relatively high occurrence of CI. This suggests that we really should give enough attention to CI after AIS. Baseline data showed that the overall patients enrolled in our study were mild. The median NIHSS score for AIS population was only 3 points. It showed that even in mild stroke, CI was a frequent consequence. And it may often masked and does not fully manifest until after acute hospitalization. So we should recommend standardized cognitive screening even in patients with mild stroke.

The study also showed that the occurrence of CI at $3 \mathrm{~m}$ and $12 \mathrm{~m}$ after stroke was significantly lower than that at $2 \mathrm{w}$ after stroke, suggesting that CI after stroke, especially early CI was partially recoverable.

Yet, indeed, for the identification of CI, the best cut-off point is still controversial. Nevertheless, MoCA or MMSE score below a cut-point is not the only accepted criteria for the Diagnosis of CI or even dementia. In this study, we defined $\mathrm{CI}$ as $\mathrm{MoCA} \leqq 22$. This is a relatively strict 


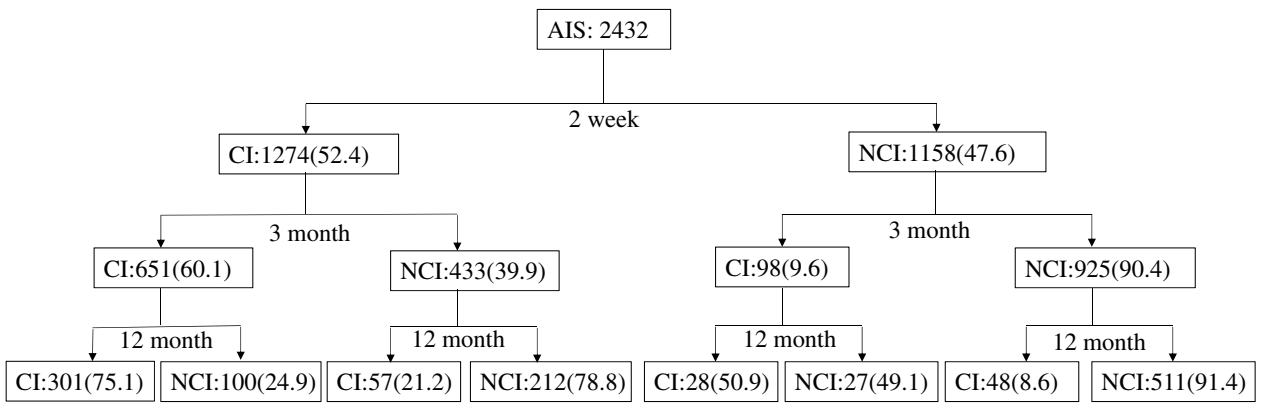

Figure 3 The longitudinal changes of cognitive impairment at each follow-up point. The figure showed the longitudinal changes of cognitive impairment at each follow-up point. This included the number of patients who actually completed the follow-up evaluation of the scale and their proportion. The number of unfinished follow-ups was not shown in the figure.

Abbreviations: $\mathrm{Cl}$, cognitive impairment; $\mathrm{NCl}$, no cognitive impairment.

definition. If calculated in terms of cognitive decline (eg MoCA<26), the proportion of abnormalities will be much higher. Cognitive decline that not severe enough to diagnose CI should also cause our attention. ${ }^{28,29}$

This study further confirmed that CI could occur both immediately after stroke and after a delay. The $\mathrm{CI}$ at different periods may have different pathogenesis, clinical features and prognosis. ${ }^{30}$ PSCI is a complex syndrome, suggesting that our future research direction should be more accurately classify people with CI after stroke in order to find a better therapeutic target. This study also analyzed the occurrence of abnormalities and recovery probability for different cognitive items. The results showed that the most easily improved cognitive item was

Table 2 Cognitive Impairment at Different Stages After AIS

\begin{tabular}{|l|l|l|l|}
\hline & At 2 Weeks n/N (\%) & At 3 Months n/N (\%) & At I2 Months n/N (\%) \\
\hline MoCA $^{8} \leqq 22$ & $1274 / 2432(52.38)$ & $749 / 2107(35.55)$ & $456 / 1335(34.16)$ \\
MoCA $^{8}<26$ & $1841 / 2432(75.70)$ & $1230 / 2107(58.38)$ & $753 / 1335(56.40)$ \\
Visuospatial/executive abnormal $^{\#}$ & $1905 / 2414(78.91)$ & $1525 / 2077(73.42)$ & $918 / 1310(70.08)$ \\
Naming abnormal & $744 / 2428(30.64)$ & $444 / 2103(21.11)$ & $248 / 1327(18.69)$ \\
Attention abnormal $^{\#}$ & $1098 / 2428(45.22)$ & $773 / 2106(36.70)$ & $445 / 1332(33.41)$ \\
Language abnormal $^{\#}$ & $1433 / 2425(59.09)$ & $1173 / 2092(56.07)$ & $740 / 1324(55.89)$ \\
Abstraction abnormal $^{\#}$ & $1359 / 2416(56.25)$ & $915 / 2048(44.68)$ & $562 / 1302(43.16)$ \\
Delayed recall abnormal $^{\#}$ & $2156 / 2413(89.35)$ & $1714 / 2057(83.33)$ & $1083 / 1308(82.80)$ \\
Orientation abnormal $^{\#}$ & $912 / 2426(37.59)$ & $491 / 2104(23.34)$ & $302 / 1334(22.64)$ \\
\hline
\end{tabular}

Notes: " MoCA indicates Montreal Cognitive Assessment. "*abnormal" is defined as the total score of this cognitive item in MoCA scale is deducted by 1 point and above.

Table 3 The Percentage of Improved Patients for Each Abnormal Cognitive Item in MoCA Scale at 3-Month (3m) and I2-Month ( $12 \mathrm{~m})$ Follow-Up

\begin{tabular}{|c|c|c|c|}
\hline & Improved $^{\&} n / N^{\#}(\%)$ (at $3 m$ vs at $\left.2 w\right)$ & Improved $^{\&} n / N^{\#}(\%)$ (at $12 m$ vs at $\left.2 w\right)$ & Improved $^{\&} n / N^{\#}(\%)$ (at $12 m$ vs at $3 m$ ) \\
\hline Visuospatial/executive & $735 / 1609(45.68)$ & $500 / 1010(49.50)$ & $308 / 908(33.92)$ \\
\hline Naming & $387 / 643(60.19)$ & $270 / 406(66.50)$ & $127 / 266(47.24)$ \\
\hline Attention & $559 / 94 I(59.40)$ & $414 / 600(69.00)$ & $25 \mathrm{I} / 452(55.53)$ \\
\hline Language & $461 / 1204(38.29)$ & $340 / 764(44.50)$ & $252 / 703(35.85)$ \\
\hline Abstraction & $519 / 1125(46.13)$ & $393 / 714(55.04)$ & $172 / 519(33.14)$ \\
\hline Delayed recall & $954 / 1814(52.59)$ & $691 / 1166(59.26)$ & $387 / 1028(37.65)$ \\
\hline Orientation & $573 / 775(73.94)$ & $388 / 506(76.68)$ & $187 / 307(60.91)$ \\
\hline
\end{tabular}

Notes: "Improved" is defined as "the total score of this cognitive item is abnormal at $2 \mathrm{w}$ or $3 \mathrm{~m}$, but increased by I point and above at a later time point ( $3 \mathrm{~m}$ or $12 \mathrm{~m}$ )". ${ }^{\#} \mathrm{~N}=$ the total number of patients who were abnormal at an earlier time point $(2 \mathrm{w}$ or $3 \mathrm{~m})$ and completed follow-up at a later time point $(3 \mathrm{~m}$ or $12 \mathrm{~m})$. ${ }_{\mathrm{n}}=\mathrm{Among} \mathrm{N}$, the number of patients who were improved at a later time point $(3 \mathrm{~m}$ or $12 \mathrm{~m})$. 
"Orientation", and the most difficultly improved cognitive item was "Language". This also helps us to more accurately understand the prognosis of different CI symptoms, and guide the subsequent evaluation focus and rehabilitation direction.

There are several limitations of our study. First, limited by the financial and human resources, we did not use a full neuropsychological battery which is more comprehensive and accurate for cognitive function evaluation. In addition to the MoCA scale, if additional assessments could be supplemented, it may offer further insight into problem areas. Second, although the overall sample size of the study was relatively large, the follow-up completion rate for $12 \mathrm{~m}$ was not very high. This will cause a sampling bias and have an impact on the relevant conclusions at this follow-up point. Third, as a national registry study, although we had selected 40 centers from different regions, we did not cover all provinces of China.

\section{Conclusion}

$\mathrm{CI}$ had a relatively high occurrence at $2 \mathrm{w}$ to $12 \mathrm{~m}$ after AIS. $\mathrm{CI}$ at $3 \mathrm{~m}$ and $12 \mathrm{~m}$ was significantly lower than that at $2 \mathrm{w}$ after stroke. The occurrence of abnormalities and recovery probability for different cognitive items also differed greatly.

\section{Data Sharing Statement}

The data that support the findings of this study are available from the corresponding author upon reasonable request.

\section{Acknowledgments}

This study is supported by grants from the Ministry of Science and Technology of the People's Republic of China (2016YFC0901001/2016YFC0901002) and grants from Beijing Municipal Science \& Technology Commission (D151100002015003).

\section{Disclosure}

The authors report no conflicts of interest in this work.

\section{References}

1. Zhou M, Wang H, Zhu J, et al. Cause-specific mortality for 240 causes in China during 1990-2013: a systematic subnational analysis for the global burden of disease study 2013. Lancet. 2016;387 (10015):251-272. doi:10.1016/S0140-6736(15)00551-6

2. GBD 2017 Causes of Death Collaborators. Global, regional, and national age-sex-specific mortality for 282 causes of death in 195 countries and territories, 1980-2017: a systematic analysis for the global burden of disease study 2017. Lancet. 2018;392:1736-1788. doi:10.1016/S0140-6736(18)32203-7
3. Lees R, Fearon P, Harrison JK, Broomfield NM, Quinn TJ. Cognitive and mood assessment in stroke research: focused review of contemporary studies. Stroke. 2012;43(6):1678-1680. doi:10.1161/STROKE AHA.112.653303

4. Fride Y, Adamit T, Maeir A, et al. What are the correlates of cognition and participation to return to work after first ever mild stroke? Top Stroke Rehabil. 2015;22(5):317-325. doi:10.1179/10749357 14Z.0000000013

5. Gaynor E, Rohde D, Large M, et al. Cognitive Impairment, Vulnerability, and mortality post ischemic stroke: a five-year follow-up of the Action on Secondary Prevention Interventions and Rehabilitation in Stroke (ASPIRE-S) cohort. J Stroke Cerebrovasc Dis. 2018;27(9):2466-2473. doi:10.1016/j.jstrokecerebrovasdis.2018. 05.002

6. Gorelick PB, Nyenhuis D. Stroke and cognitive decline. JAMA. 2015;314(1):29-30. doi:10.1001/jama.2015.7149

7. Winstein CJ, Stein J, Arena R, et al. Guidelines for adult stroke rehabilitation and recovery: a guideline for healthcare professionals from the American Heart Association/American Stroke Association. Stroke. 2016;47(6):e98-e169. doi:10.1161/STR.0000000000000098

8. Liu M, Wu B, Wang WZ, Lee LM, Zhang SH, Kong LZ. Stroke in China: epidemiology, prevention, and management strategies. Lancet Neurol. 2007;6:456-464. doi:10.1016/S1474-4422(07)70004-2

9. Wang W, Jiang B, Sun H, et al. Prevalence, incidence, and mortality of stroke in China: results from a nationwide population-based survey of 480687 adults. Circulation. 2017;135(8):759-771. doi:10.1161/ CIRCULATIONAHA.116.025250

10. Wang Y, Jing J, Meng X, et al. The Third China National Stroke Registry (CNSR-III) for patients with acute ischaemic stroke or transient ischaemic attack: design, rationale and baseline patient characteristics. Stroke Vasc Neurol. 2019;4(3):158-164. doi:10. 1136/svn-2019-000242

11. Stroke-1989. Recommendations on stroke prevention, diagnosis, and therapy. Report of the WHO task force on stroke and other cerebrovascular disorders. Stroke. 1989;20(10):1407-1431. doi:10.1161/01. STR.20.10.1407

12. Hurford R, Li L, Lovett N, Kubiak M, Kuker W, Rothwell PM, Oxford Vascular Study. Prognostic value of "tissue-based" definitions of TIA and minor stroke: population-based study. Neurology. 2019;92 (21):e2455-e2461. doi:10.1212/WNL.0000000000007531

13. Nasreddine ZS, Phillips NA, Bédirian V, et al. The montreal cognitive assessment, MoCA: a brief screening tool for mild cognitive impairment. J Am Geriatr Soc. 2005;53:695-699. doi:10.1111/j.15325415.2005.53221.x

14. Salvadori E, Pasi M, Poggesi A, Chiti G, Inzitari D, Pantoni L. Predictive value of MoCA in the acute phase of stroke on the diagnosis of mid-term cognitive impairment. J Neurol. 2013;260 (9):2220-2227. doi:10.1007/s00415-013-6962-7

15. Lees R, Selvarajah J, Fenton C, et al. Test accuracy of cognitive screening tests for diagnosis of dementia and multidomain cognitive impairment in stroke. Stroke. 2014;45(10):3008-3018. doi:10.1161/ STROKEAHA.114.005842

16. Zuo L, Dong Y, Zhu R, et al. Screening for cognitive impairment with the montreal cognitive assessment in Chinese patients with acute mild stroke and transient ischaemic attack: a validation study. $B M J$ Open. 2016;6(7):e011310. doi:10.1136/bmjopen-2016-011310

17. Wen HB, Zhang ZX, Niu FS, Li L. The application of montreal cognitive assessment in urban Chinese residents of Beijing. Zhonghua Nei Ke Za Zhi. 2008;47:36-39.

18. Buysse DJ, Reynolds CF, Monk TH, Berman SR, Kupfer DJ. The pittsburgh sleep quality index: a new instrument for psychiatric practice and research. Psychiatry Res. 1989;28(2):193-213. doi:10.1016/0165-1781(89)90047-4

19. Johns MW. A new method for measuring daytime sleepiness: the epworth sleepiness scale. Sleep. 1991;14(6):540-545. doi:10.1093/ sleep/14.6.540 
20. Kroenke K, Spitzer RL, Williams JBW. The PHQ-9: validity of a brief depression severity measure. J Gen Intern Med. 2001;16 (9):606-613. doi:10.1046/j.1525-1497.2001.016009606.x

21. Spitzer RL, Kroenke K, Williams JB, Löwe B. A brief measure for assessing generalized anxiety disorder: the GAD-7. Arch Intern Med. 2006;166(10):1092-1097. doi:10.1001/archinte.166.10.1092

22. Peters DM, Fritz SL, Krotish DE. Assessing the reliability and validity of a shorter walk test compared with the 10-meter walk test for measurements of gait speed in healthy, older adults. $J$ Geriatr Phys Ther. 2013;36(1):24-30. doi:10.1519/JPT.0b013 e318248e20d

23. Duncan PW, Lai SM, Bode RK, Perera S, DeRosa J. Stroke impact scale-16: a brief assessment of physical function. Neurology. 2003;60 (2):291-296. doi:10.1212/01.WNL.0000041493.65665.D6

24. Jacquin A, Binquet C, Rouaud O, et al. Post-stroke cognitive impairment: high prevalence and determining factors in a cohort of mild stroke. J Alzheimers Dis. 2014;40(4):1029-1038. doi:10.3233/JAD131580

25. Sun JH, Tan L, Yu JT. Post-stroke cognitive impairment: epidemiology, mechanisms and management. Ann Transl Med. 2014;2 (8):80-83. doi:10.3978/j.issn.2305-5839.2014.08.05
26. Yu KH, Cho SJ, Oh MS, et al. Cognitive impairment evaluated with vascular cognitive impairment harmonization standards in a multicenter prospective stroke cohort in Korea. Stroke. 2013;44 (3):786-788. doi:10.1161/STROKEAHA.112.668343

27. Zhou DH, Wang JY, Li J, Deng J, Gao C, Chen M. Frequency and risk factors of vascular cognitive impairment three months after ischemic stroke in china: the Chongqing stroke study. Neuroepidemiology. 2005;24(1-2):87-95. doi:10.1159/000081055

28. Langa KM, Levine DA. The diagnosis and management of mild cognitive impairment: a clinical review. JAMA. 2014;312 (23):2551-2561. doi:10.1001/jama.2014.13806

29. Chaurasia RN, Sharma J, Pathak A, Mishra VN, Joshi D. Poststroke cognitive decline: a longitudinal study from a tertiary care center. J Neurosci Rural Pract. 2019;10(03):459-464. doi:10.1055/s-00391697872

30. Gottesman RF, Hillis AE. Predictors and assessment of cognitive dysfunction resulting from ischaemic stroke. Lancet Neurol. 2010;9 (9):895-905. doi:10.1016/S1474-4422(10)70164-2

\section{Publish your work in this journal}

Neuropsychiatric Disease and Treatment is an international, peerreviewed journal of clinical therapeutics and pharmacology focusing on concise rapid reporting of clinical or pre-clinical studies on a range of neuropsychiatric and neurological disorders. This journal is indexed on PubMed Central, the 'PsycINFO' database and CAS, and is the official journal of The International Neuropsychiatric Association (INA). The manuscript management system is completely online and includes a very quick and fair peer-review system, which is all easy to use. Visit http://www.dovepress.com/testimonials.php to read real quotes from published authors. 